\title{
Composition and Thickness Mapping Using STEM EDS
}

\author{
Meiken Falke ${ }^{1}$, Johanna Kraxner ${ }^{2}$, Ralf Terborg ${ }^{1}$, Gerald Kothleitner ${ }^{2}$ and Werner Grogger ${ }^{2}$ \\ 1. Bruker Nano GmbH, Berlin, Germany. \\ 2. Institute for Electron Microscopy and Nanoanalysis (FELMI) \& \\ Graz Centre for Electron Microscopy (ZFE), Graz University of Technology, Graz, Austria.
}

SDD technology enabled high take-off angle and high collection angle EDS detector arrangements. Such detectors in combination with high brightness electron sources made EDS a powerful analysis tool for bulk- and electron transparent samples. In STEM-mode, where EELS is an established spectroscopy technique, EDS is now accepted as a useful complementary analysis method [1] and in many cases as an alternative to EELS element mapping.

For quantitative analysis of STEM EDS data, there are two main options available, the Cliff-Lorimer and the Zeta-factor method [2]. The latter delivers a more complete picture of the sample. It offers the correction for absorption effects by taking the specimens mass thickness into account, while the CliffLorimer method avoids thickness effects by using relative Cliff-Lorimer factors, for the sake of simplicity of data acquisition and analysis. Both options have their pros and cons. If EDS is used alternatively to EELS though, retrieving the sample thickness from EDS may be a valuable addition to obtainable results. HAADF data could been used for thickness determination or verification too, if no other, e.g. remaining diffraction, effects obscure this analysis. Also, using the EDS background has been suggested. We report on determining the sample thickness map using the Zeta-factor approach.

The number of characteristic X-ray counts is proportional to the number of atoms per unit volume, the respective ionization cross section, fluorescence yield, geometrical collection efficiency (solid angle), detection quantum efficiency and the number of incident electrons [3]. This means, that, if enough of these parameters are available, e.g. via calibration using standards, the other unknown values can be determined either directly or via a set of equations or additionally using an iterative process [2]. For standard-less analysis atomic data must be well known [4]. Several flavors of standard based approaches have been realized. Element specific Zeta-factors, once determined relying on one or several standards for a given data acquisition geometry, allow absorption corrected composition mapping and mass thickness $(\rho t)$ retrieval for an unknown specimen investigated in the same geometry, if the beam current is known. Thickness ( $\left.t_{\mathrm{EDS}}\right)$ maps can be calculated from the same data set by calibrating the mass thickness using a density ( $\rho_{\text {EDS }}$ ) value, which must be either known or can be estimated (with care) from the element composition found by EDS. $\mathrm{A} \mathrm{TiO}_{2}$ lamella investigated by EELS and EDS in comparison is used to demonstrate this approach. Measurement conditions: FEI Titan SuperX, $300 \mathrm{kV}, 270 \mathrm{pA}$ beam current, $0.17 \mathrm{sr}$ solid angle (single quadrant), no window, dwell time per pixel: $150 \mathrm{~ms}$.

Fig. 1a) shows the HAADF image, the $t / \lambda$ map obtained from EELS data, the EDS composition map and the EDS thickness map retrieved from a Hyperspectral data set of $\mathrm{TiO}_{2}$ knowing the beam current and having determined Zeta-factors before this analysis using the marked 400 pixel by 40 pixel sample region as standard. Any suitable separate standard could of course be used as well. To obtain $t_{\mathrm{EDS}}$ the density $\rho_{\mathrm{EDS}}=2.07 \mathrm{~g} / \mathrm{cm}^{3}$ was calculated from the $\mathrm{TiO}_{\mathrm{x}}$ composition, found by EDS. Other materials including $\mathrm{Al}_{2} \mathrm{O}_{3}$ and $\mathrm{SiN}_{\mathrm{x}}$ were analysed by EELS and EDS in comparison in the same way achieving similar results. All map types reproduce roughly the same thickness distribution ranging between 
100 and $130 \mathrm{~nm}$. The $\mathrm{t} / \lambda$ data are less noisy than the mass thickness data retrieved from EDS. One reason is, that in EELS nearly all electrons are collected while EDS, at $\sim 0.17$ sr solid angle (just one of four $30 \mathrm{~mm}^{2}$ superX quadrants was used), only captures a part of the ionization events. Suitable binning and/or longer measurement times and a higher collection angle, e.g. using all 4 SuperX quadrants, will be helpful to increase statistics in case of EDS. The data analysis shows, that EDS using the Zeta-factor method performs well and delivers useful information including the sample thickness distribution.

References:

[1] G. Kothleitner et al, Microsc. Microanal. 20 (2014) p. 678.

[2] M. Watanabe \& D B Williams, J. of Micr. 221 (2006) p. 89.

[3] R. F. Egerton \& S. C. Cheng, Ultramicroscopy 55 (1994) p. 43.

[4] R. Gauvin, Microsc. Microanal. 18 (2012) p. 915.
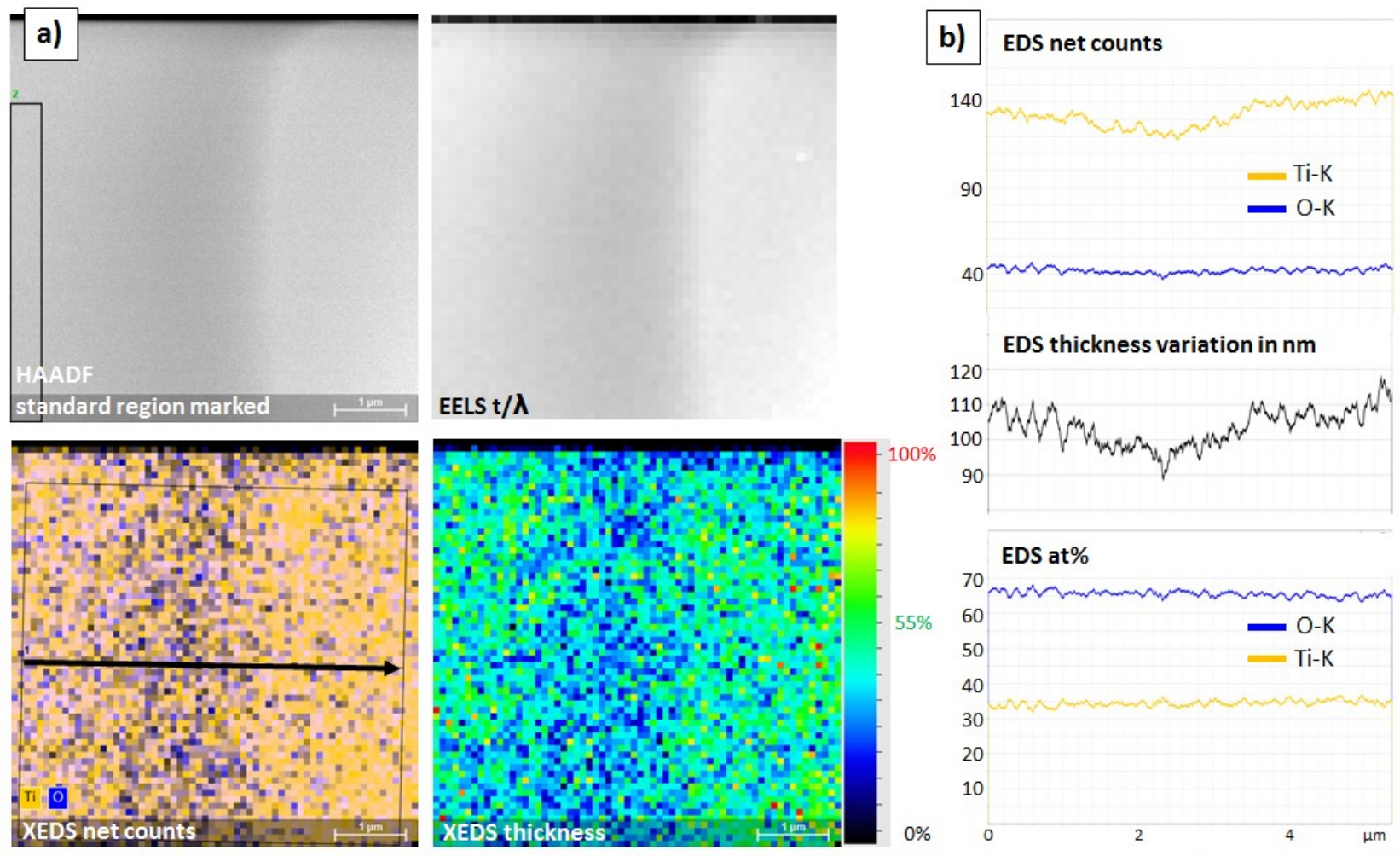

Figure 1. a) HAADF with region used as Zeta-factor standard marked, EELS $t / \lambda$ map using $10 x 10$ binning of a $512 \times 512$ spectrum image, the respective EDS 512x512 net count map with a broadened line scan marked and the EDS thickness map of $\mathrm{TiO}_{2}$ using $8 \times 8$ binning. The thickness determined by EELS in the line scan region varies between $110 \mathrm{~nm}$ and $125 \mathrm{~nm}$. These absolute values were calculated from the $\mathrm{t} / \lambda$ map with $\lambda=140 \mathrm{~nm}$ for $\mathrm{TiO}_{2}$, which was experimentally determined using a FIB rod of known composition and diameter [1]. The noisier EDS thickness map was calibrated using $\rho_{\text {EDS }}=2.07 \mathrm{~g} / \mathrm{cm}^{3}$, calculated from the element composition found by EDS. The respective thickness values for the marked line scan are shown on the right in b). b) Profiles of EDS net counts, thickness and quantified element distribution along the line as marked in the EDS net count image. More net counts for Ti-K appear in thicker regions while the O-K signal suffers from absorption. The absorption corrected data at $\%$ in show the correct $\mathrm{TiO}_{2}$ composition over the whole broadened line scan. 